\title{
Droplet Clusters: Exploring the Phase Space of Soft Mesoscale Atoms
}

\author{
Jan Guzowski* and Piotr Garstecki ${ }^{\dagger}$ \\ Institute of Physical Chemistry, Polish Academy of Sciences ul. Kasprzaka 44/52, 01-224 Warsaw, Poland \\ (Received 4 September 2014; revised manuscript received 10 March 2015; published 6 May 2015; corrected 25 March 2016)
}

\begin{abstract}
We report three-dimensional structures-mesoscale "atoms"- comprising up to $N=8$ aqueous droplets compressed in a liquid shell. In contrast to hard colloids that self-assemble into structures unique for a given $N$, we observe multiple metastable states. We attribute this unexpected richness of metastable structures to the deformability of the cores that introduces irreducible many-body interactions between the droplets. These exotic, often highly anisotropic, structures are locally stable. The structures displaying highly nonoptimum packing - and hence interfacial energy much higher than that of the lowest-energy stateexhibit finite energy barriers that prevent restructuring and relaxation of energy.
\end{abstract}

DOI: 10.1103/PhysRevLett.114.188302

PACS numbers: 82.70.Dd, 47.55.D-, 61.46.Bc, 83.50.Ha

In this Letter we show that introduction of an additional degree of freedom into the assembly of small clusters of particles brings in a richness of structures. Deformability of the particles results in irreducible many-body interactions that complicate the energy landscape and create multiple local minima associated with exotic metastable "atoms." Generation of colloidal atoms is of high interest for their applications in the design of new materials [1] via their selfassembly into superstructures analogous to macromolecules [2,3], ordered 2D lattices [4,5], or fully periodic 3D lattices [6], the latter being particularly useful as potential templates for the synthesis of photonic band gap [7] or porous materials $[4,8]$. Wang et al. [9] recently proposed a fascinating example-particles with spots arranged in accord with well-defined point-group symmetries. DNA functionalization of the spots yielded atoms presenting "valences" and assembling into colloidal "molecules".

Wang et al. fabricated $N$-valent particles via capillary self-assembly of $N$ spherical particles close packed in an evaporating droplet-a method proposed by Manoharan et al. [10]. It is known that such close-packed structures minimize the second moment of mass and are unique for a given $N$ (for $N \leq 11$ ). Lauga and Brenner [11] explained this uniqueness by minimization of the surface energy under constraints imposed by rigidity of the particles. The final structures being at equilibrium warrants high yield, yet it also limits the range of available architectures. Recently, Arkus et al. [12] and Meng et al. [13] studied self-assembly of spheres via short-range attractions and revealed the existence of multiple ground states observed with different probabilities [13]. Accordingly, to date, two types of systems have been reported: (i) those always presenting

Published by the American Physical Society under the terms of the Creative Commons Attribution 3.0 License. Further distribution of this work must maintain attribution to the author(s) and the published article's title, journal citation, and DOI. the same structure of the atom for a given $N$ or (ii) various morphologies and topologies of the atoms, without the possibility to control their occurrence.

Here we show that the use of soft cores (droplets) introduces several new effects. Deformability of drops replaces pair with many-body interactions, such that the structure of the atom becomes a function of deformation of all the cores. This produces a rich variety of accessible states.

Interestingly, capillary forces generate energy barriers trapping noncompact metastable structures. The occurrence of the structures can be tuned by the volume fraction $\phi=V_{c} /\left(V_{c}+V_{s}\right)$, where $V_{c}$ and $V_{s}$ are the volume of the cores and of the shell. Such control has not been available in the assembly of hard colloids.

We used three immiscible liquids supplied under constant rates of flow to generate double droplets comprising an oil shell and multiple aqueous cores [14]. First, at a $T$ junction the aqueous (core) stream (water dyed with methylene blue) broke into droplets in the (shell) hydrocarbon phase $(1 \% \mathrm{w} / \mathrm{w}$ solution of SPAN80 in $7: 3 \mathrm{w} / \mathrm{w}$ mixture of PMX 200 silicone oil and hexadecane). The chain of aqueous plugs (the volume polydispersity of those plugs was around 3\%) flowed in a narrow channel $(150 \mu \mathrm{m} \times 150 \mu \mathrm{m})$ and broke into segments upon entering a wide chamber $(1.5 \mathrm{~mm} \times 1.5 \mathrm{~mm})$ with the third fluorocarbon fluid (3M Novec 7500) via a mechanism similar to step emulsification [15]. Such generated double droplets relaxed to their final morphologies under capillary forces.

Generation and self-assembly of double droplets was controlled by the geometry of the channels, the rates of flow, and also the tilt angle $\psi$ with respect to gravity. $\psi$ controlled the number $N$ of the aqueous cores independently of $\phi$. Importantly, buoyancy was not strong enough to deform structures (the Bond number $\approx 0.6[16]$ ), making our classification of emulsion atoms valid also for smaller droplets, possibly down to microns in size. The role of the 
surfactant (SPAN 80) in the shell phase was to lower the core-shell interfacial tension, cause spontaneous wetting of the cores by the shell liquid, and prevent coalescence of the cores by separating them with surfactant films (those possibly being bilayers $[17,18])$. We measured the contact angle between the cores to be $\alpha=45 \pm 5^{\circ}$ (inset of Fig. 1).

$(\phi, N)$ phase diagram.-For any given $N$, there was a threshold volume of the shell liquid, below which the multiple droplets spontaneously formed well-defined, compact structures. The threshold value of the volume fraction can be derived from the maximum volume fraction $\phi_{\max }(N)=N a^{3} / R_{\min }(N)^{3}$ of $N$ hard spheres of radius $a$ in a sphere of radius $R_{\min }(N)$. The aqueous cores, due to the finite contact angle $\alpha$ between them, pack more tightly than hard spheres. Their densest possible packing is $\phi_{\text {packing }}(N)=[1+\Delta(N, \alpha)] \phi_{\max }(N)$, where $\Delta(N, \alpha)$ is a small positive function [see Supplemental Material (SM) [20]].

The topologies of structures obtained at $\phi$ just above $\phi_{\text {packing yet }}<\phi_{\text {capillary }}$ [Fig. 1(a)] show the solutions of the purely mathematical problem of densest packing of $N$ spheres in a sphere [19], where $\phi_{\text {capillary indicates the }}$ experimental cases with 5\% asphericity of the shell. For $\phi_{\text {capillary }}<\phi<\phi_{\text {chain }}$, we observed multiple close-packed structures [Fig. 1(b)] different from the in-sphere packings [19], with the shell significantly deformed from the spherical

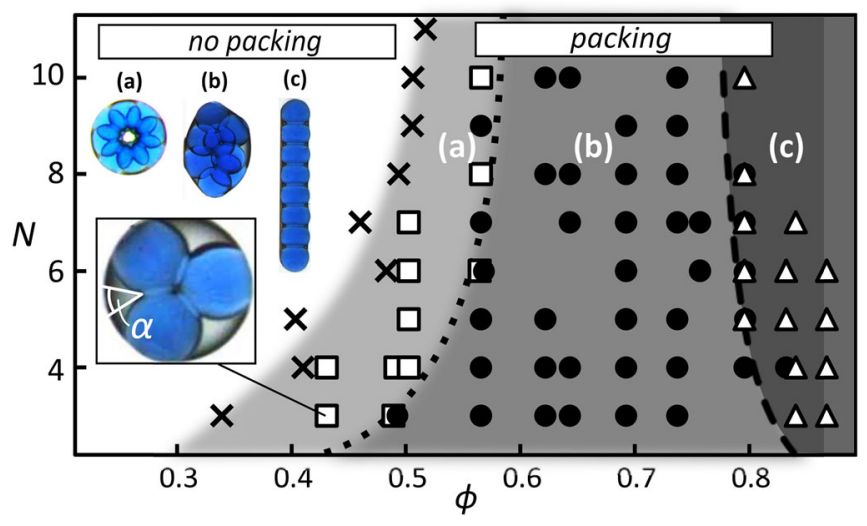

FIG. 1 (color online). Stability diagram for droplets comprising $N$ cores in a shell at volume fraction $\phi$ of the cores. The crosses show numerical results for $\phi_{\text {packing }}$ : the densest packing of $N$ hard spheres inside a sphere after Pfoertner [19], modified in order to account for partial overlap of the spheres (the equilibrium contact angle between the drops was $\alpha=45 \pm 5^{\circ}$, as shown in the inset for the case $N=3$ ). The cores close pack only for $\phi>\phi_{\text {packing. }}$. The dotted line is a guide to the eye approximating $\phi_{\text {capillary }}$, above which the capillary deformation of the shell impacts the structures. The dashed line is given by Eq. (1) with $\phi_{\text {chain, } \infty}=$ $0.76 \pm 0.02$. The symbols in the "packing" regime show experimental results for (a) negligible deformation of the shell $(<5 \%$ asphericity, squares), (b) strong deformation of the shell (>5\% asphericity, circles), and (c) linear chains (triangles). Micrographs show representatives of these categories for $N=8$. shape ( $>5 \%$ asphericity). For $\phi>\phi_{\text {chain }}$, we observed stable linear chains of droplets connected by capillary bridges of the shell phase [Fig. 1(c)], in which the droplets did not rearrange into more compact structures. Our observations suggest that the critical value $\phi_{\text {chain }}$, above which the chains form, decreases with $N$. This can be explained as follows. In the linear configuration the volume fraction is $\phi=\left[1+(N-1) V_{s 1} /\left(N V_{c 1}\right)\right]^{-1}$, where $V_{s 1}$ and $V_{c 1}$ are the volumes of a single bridge and a single core, respectively. When $V_{s 1}$ exceeds a critical value $V_{s 1 \text {,crit }}$, the neighboring bridges merge and the chain folds into a 3D structure. This corresponds to a critical value of $\phi$ :

$$
\phi_{\text {chain }}(N)=\phi_{\text {chain }, \infty}+\left(V_{s 1, \text { crit }} / V_{c 1}\right) N^{-1},
$$

where $\phi_{\text {chain, } \infty}=\left[1+V_{s 1, \text { crit }} / V_{c 1}\right]^{-1}$. From a fit to the data in Fig. 1 (dashed line), we find $\phi_{\text {chain, } \infty}=0.76 \pm 0.02$.

In the regime of $\phi$ between $\phi_{\text {packing }}$ and $\phi_{\text {chain }}$, we observed different final structures with the probability of observing each of them depending on $\phi$. The general trend was that the most probable structures became more compact ones as $\phi$ decreased. In Fig. $2(N=3, \ldots, 7)$ and Fig. S2 of the Supplemental Material [20] $(N=8)$, we list all the observed structures along their most probable volume fraction $\bar{\phi}$. We determined the latter as $\bar{\phi}=\bar{\phi}_{i}=\sum_{\phi} \phi n_{i, \phi} / \sum_{\phi} n_{i, \phi}$, in which the summation runs over all experimentally available $\phi$ and where $n_{i, \phi}$ is the counted number of occurrences of the structure $i$ at a given $\phi$.

Richness of structures as compared to self-assembly of hard spheres.-In the simplest cases of $N=3$ and $N=4$, apart from the linear structures, we observed the same configurations (a triangle for $N=3$ and a tetrahedron for $N=4$ ) as Manoharan et al. [10]. Starting from $N=5$, besides the structures minimizing the second moment of mass, we observed a rich variety of droplet architectures that extends the list of structures that can be assembled from spherical objects by capillary forces. There are several reasons for the stability of noncompact structures. In contrast to evaporating droplets, where the final volume fraction of the particles is 1 , our system allows us to tune $\phi$. Second, deformability of the droplets introduces additional elastic interactions and energy barriers for rearrangement [21]. The increased stability with a lack of effective thermalization mechanism allows for a rich variety of topologies and geometries of the 3D assemblies.

Comparison with minimal clusters of "sticky" spheres.-We identified most of the structures as the ground states of packings of sticky spheres (i.e., interacting via short-ranged attraction). These packings [12] maximize the number of contacts. In our experiment for $N=4, \ldots, 7$, we identified the structures classified by Arkus et al. except the one of $C_{3 v}(N=7)$ structures characterized by a particularly large second moment of mass. This structure (not shown in Fig. 2) develops multiple concave regions that are likely to be penalized energetically. Interestingly, at 

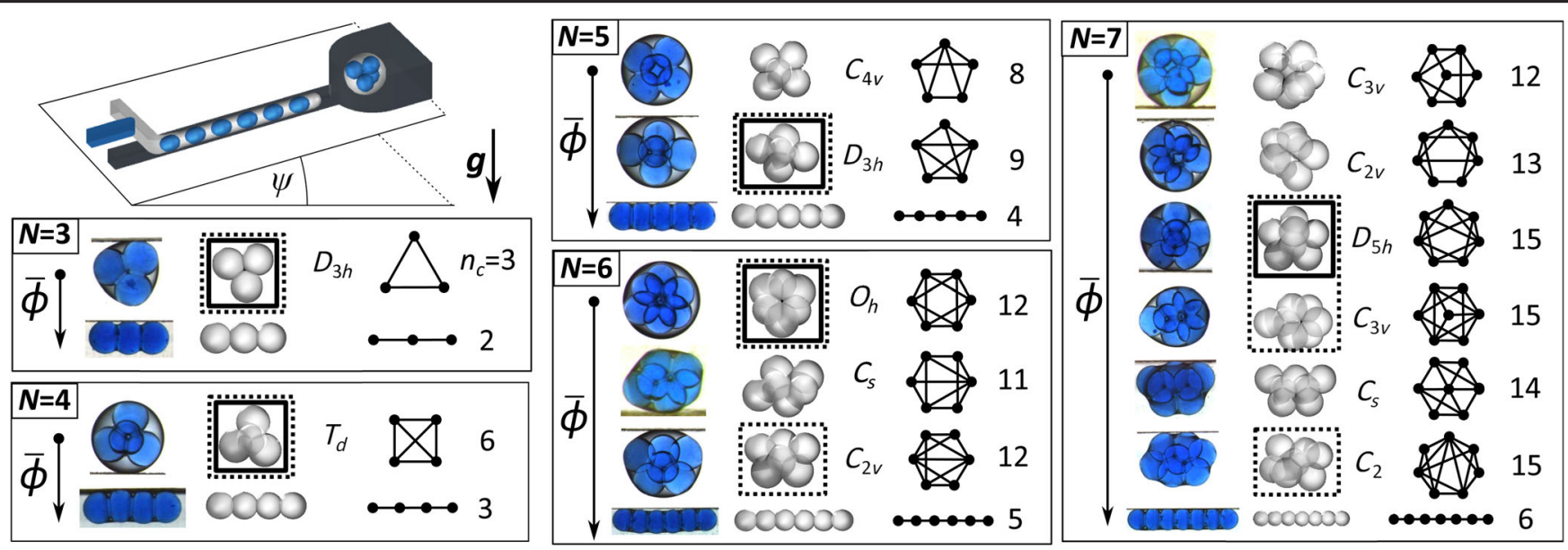

FIG. 2 (color online). Microfluidic device with two $T$ junctions. Multiple droplets form by breakup of oil (light gray) containing aqueous cores (blue) at the inlet to the chamber filled with a third immiscible fluorocarbon phase (dark gray). The structures for each $N$ are ordered by their most probable volume fraction $\bar{\phi}(N=8$ in SM [20]). The point-group symmetries are given in Schoenflies notation. Solid frames indicate structures minimizing second moment of mass, dotted ones indicate maximum number of contacts $n_{c}$. The diameter of the aqueous cores ranges from $200 \mu \mathrm{m}$ (smallest $\phi$ ) to $400 \mu \mathrm{m}$ (largest $\phi$, linear chains). In the case of structure $D_{5 h}$ for $N=7$, the value of $n_{c}$ could not be determined due to sensitive relation to $\alpha$ : we assumed $n_{c}$ to be the same as for nonoverlapping spheres, possibly underestimating the value by 1 .

$\phi \approx \phi_{\text {packing }}$ we observed three new structures $\left[C_{s}(N=6)\right.$, $\left.C_{s}(N=7), C_{2 v}(N=7)\right]$ that neither maximized the number of contacts nor could be identified as the in-sphere packings. In the SM [20] we list analogical observations for the case $N=8$.

Volume fraction as an additional control parameter.Figure 3 shows conditional probabilities $P=P_{i}=$ $P(i \mid N)=n_{i} / \sum_{j} n_{j}$ of occurrence of the structures (for $N=7$ or $N=8$ ) within fixed $\phi$ intervals (for each interval $\left.\sum_{i} P_{i}=1\right)$. We also calculated the second moment of mass $M_{2}$ and the maximum length $D_{\max }$ of a straight interval that
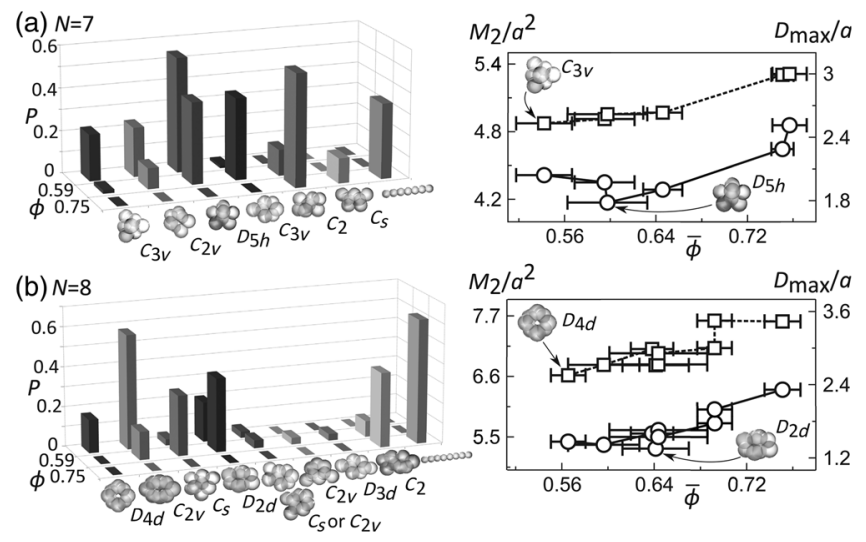

FIG. 3. Probabilities $P$ of occurrence of different "atoms" at different applied volume fractions $\phi$ for $N=7$ (a) and $N=8$ (b) (for experimental snapshots, see SM [20]). The structures $C_{s}$ and $C_{2 v}$ for $N=8$ were indistinguishable in the experiment and were counted as one. The graphs show the second moment of mass $M_{2}$ (circles, left axes) and the maximum length $D_{\max }$ (squares, right axes) as functions of $\bar{\phi}$. The horizontal error bars are the standard deviations of the corresponding probability distributions. can be inscribed into the structure. The structures that minimize $D_{\max }$ (e.g., $C_{3 v}$ and $D_{4 d}$ at $N=7$ and $N=8$, respectively) are in fact the densest in-sphere packings; i.e., they also minimize the radius of the circumsphere. The general trend is that $D_{\max }$ monotonically increases with $\phi$, which suggests that $D_{\max }$ could be treated as a relevant measure of the compactness of the structures. On the other hand, $M_{2}$ develops a minimum at intermediate $\phi$, corresponding to the structures $D_{5 h}$ at $N=7$ and $D_{2 d}$ at $N=8$, the ones observed previously by Manoharan et al. These examples show that tuning $\phi$ provides for a level of control of the geometric characteristics of the structures.

Discussion.-We illustrate the unexpected observation that capillary forces can stabilize highly noncompact shapes of liquid droplets with the simple case of three drops and two structures: a (compact) triangle and a (noncompact) linear chain. We used the Surface Evolver to calculate the energy $E(\beta)$ parametrized by the dihedral angle $\beta$ (Fig. 4; see also SM [20]). We assumed high $\phi$ $(>0.76)$ such that in the linear chain the oil forms capillary bridges. We modeled these bridges by attractive potentials $V_{i j}=f r_{i j}$ between droplets $\{i, j\}=\{1,2\}$ and $\{2,3\}$, with $f$ being a constant force (we took $f=\gamma_{\text {core }} a$, where $a$ is the radius of an undeformed droplet) and $r_{i j}$ the separation of centers of mass of the drops. At $\beta=\beta_{\text {crit }}$ the bridges merge (we assume $\beta_{\text {crit }}=45^{\circ}$ ). We modeled this by adding to $E(\beta)$ the term $V_{13}=\Theta\left(\beta-\beta_{\text {crit }}\right) f\left[r_{13}-r_{13}\left(\beta_{\text {crit }}\right)\right]$, with $\Theta$ being the Heaviside step function. The resulting energy landscape develops two local minima at $\beta=0$ and $\beta=120^{\circ}$, separated by an energy barrier of the order $10^{-1} \gamma_{\text {core }} a^{2}$. The presence of the minimum at $\beta=0$ can be traced back to nonmonotonicity of the deformation of a droplet subjected 


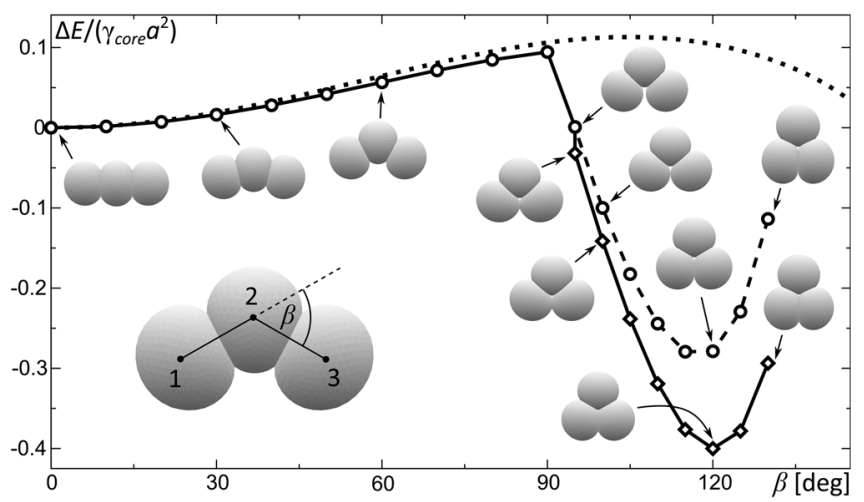

FIG. 4. Energy landscape of a cluster of $N=3$ droplets calculated by replacing capillary bridges with attractive potentials. Dashed line indicates a metastable branch corresponding to situation without a surfactant bilayer between droplets 1, 3 . Formation of the bilayer shifts the system to the globally stable branch (solid line). The dotted line corresponds to an approximate analytical expression [22] (see also SM [20]) assuming point forces acting on droplet 2 (instead of droplets 1, 3).

to a point force (expressed as a function of the angular distance from the point force; see also SM [20]) and stabilizes chains of droplets squeezed by capillary bridges. A similar mechanism of "capillary jamming" may be responsible for stabilization of other noncompact structures observed in the experiment. In addition, relaxation of structures might be to some extent hindered by formation of sticky surfactant bilayers. In order to estimate the importance of the latter, one would have to repeat the experiment using nonsticky droplets.

In our experiments buoyancy tends to flatten the structures against the upper wall of the chamber. In our previous work [21] exploiting the same set of fluids, we observed the formation of 2D structures when $D_{\max }$ approached $1.5 \mathrm{~mm}$. Here, we generated at least twice smaller droplets $\left(D_{\max }<0.7 \mathrm{~mm}\right.$, excluding the chains) and observed 3D structures with full 3D symmetry. Thus, we a posteriori conclude that the presence of the wall and generation of droplets on demand [21] or by using buoyancy did not affect the final structures. We note that the same structures can be obtained with the use of a passive coflow breakup mechanism. Indeed, packing of droplets in double emulsions have also been previously observed by Kim et al. [23] who used concentric capillaries. The authors synthesized solid particles by UV curing the multiple droplets. In combination with this or similar solidification techniques, our work could be used to produce particles with pointgroup symmetry corresponding to any of the available metastable states.

We expect the variety of structures and their $\phi$ dependence to remain valid whenever the system is dominated by capillary forces and low Reynolds number hydrodynamics. Even at the scale of single microns, the typical energy barrier $10^{-1} \gamma_{\text {core }} a^{2}$ ( $\gamma_{\text {core }}$ being of the order $1 \mathrm{mN} / \mathrm{m} \mathrm{[21])}$ is of the order $10^{4} k_{B} T$, so that capillary trapping of the metastable states should remain effective. Generation of micron-sized droplets (including multiple droplets) is in reach of current microfluidic techniques [24]. Sorting of the structures could possibly be done inside microchannels by exploiting the shape dependence of the hydrodynamic drag or by some kind of active sorting [25]. Otherwise, by improving flow control one could perhaps narrow the probability distributions eliminating completely the necessity of sorting. More research is needed to determine the dependence of structures on the ratio $\gamma_{\text {core }} / \gamma_{\text {shell }}$, which in our case was around 4 (for actual values of surface tensions see Ref. [21]). Presumably this number should be larger than unity but not too large in order to allow for metastable states (as resulting from competition between core and shell interfacial energies).

In summary, we reported assembly of deformable cores into a variety of $3 \mathrm{D}$ assemblies. We found that the structures depend on $\phi$. At low $\phi$, i.e., at $\phi$ only slightly larger than $\phi_{\text {packing }}$, yet smaller than $\phi_{\text {capillary }}$, the shell and cores remain nearly undeformed. The cores freely rearrange to minimize the deformation of the shell which leads to the structures corresponding to the densest possible packing of spheres in a sphere. At intermediate $\phi$, i.e., at $\phi_{\text {capillary }}<\phi<\phi_{\text {chain }}$, the shell is significantly deformed and the capillary forces become important. The capillary energy barriers allow for observation of multiple metastable structures. Finally, at $\phi>\phi_{\text {chain }}$ the shell forms capillary bridges between the cores preventing rearrangement and stabilizing linear arrays of droplets. We can increase the yield of the chosen types of structures by fine-tuning $\phi$, which we achieve by adjusting the rates of flow of the immiscible liquids. These results could serve as guideline, e.g., for synthesis of particles or for printing of tissuelike materials [26]. Finally, our work provides a first experimental example of a system with repulsive "capillary interactions" between capillary monopoles [27], which in our case are associated with the contact forces between drops. In a wider perspective, this repulsion, by promoting linear structures, might also have an effect on ordering and jamming in emulsions [28].

Research performed within the Iuventus Plus IP2011/ 016971 and IP2012/012472 grants from the Ministry of Science and Higher Education and within the European Research Council Starting Grant No. 279647. J. G. acknowledges financial support from Polish Ministry of Science under grant Mobility Plus 1058/MOB/2013/0.

*jguzowski@ichf.edu.pl garst@ichf.edu.pl

[1] F. Li, D. P. Josephson, and A. Stein, Angew. Chem., Int. Ed. Engl. 50, 360 (2011).

[2] D. Zerrouki, J. Baudry, D. Pine, P. Chaikin, and J. Bibette, Nature (London) 455, 380 (2008). 
[3] Q. Chen, J. K. Whitmer, S. Jiang, S. C. Bae, E. Luijten, and S. Granick, Science 331, 199 (2011).

[4] Q. Chen, S. C. Bae, and S. Granick, Nature (London) 469, 381 (2011).

[5] Q. Chen, S. C. Bae, and S. Granick, J. Am. Chem. Soc. 134, 11080 (2012).

[6] Z. Quan and J. Fang, Nano Today 5, 390 (2010).

[7] S.-H. Kim, S. Y. Lee, S.-M. Yang, and G.-R. Yi, NPG Asia Mater. 3, 25 (2011).

[8] K. Miszta et al., Nat. Mater. 10, 872 (2011).

[9] Y. Wang, Y. Wang, D. R. Breed, V. N. Manoharan, L. Feng, A. D. Hollingsworth, M. Weck, and D. J. Pine, Nature (London) 491, 51 (2012).

[10] V. N. Manoharan, M. T. Elsesser, and D. J. Pine, Science 301, 483 (2003).

[11] E. Lauga and M. P. Brenner, Phys. Rev. Lett. 93, 238301 (2004).

[12] N. Arkus, V. N. Manoharan, and M. P. Brenner, Phys. Rev. Lett. 103, 118303 (2009).

[13] G. Meng, N. Arkus, M. P. Brenner, and V. N. Manoharan, Science 327, 560 (2010).

[14] We used pressurized containers and hydraulic resistors of very large resistance which provided more stable flow rates as compared to those provided by pumps.

[15] R. Dangla, E. Fradet, Y. Lopez, and C. N. Baroud, J. Phys. D 46, 114003 (2013).

[16] In calculation of the Bond number, we used the core-shell interfacial tension (as the main factor holding the structure against buoyancy) and assumed the size of the structure to be $0.5 \mathrm{~mm}$.

[17] N. D. Denkov, S. Tcholakova, K. Golemanov, and A. Lips, Phys. Rev. Lett. 103, 118302 (2009).

[18] A. R. Thiam, N. Bremond, and J. Bibette, Langmuir 28, 6291 (2012).

[19] H. Pfoertner, http://www.randomwalk.de/sphere/insphr/ spisbest.txt.

[20] See Supplemental Material at http://link.aps.org/ supplemental/10.1103/PhysRevLett.114.188302 for experimental results for $N=8$ and the details of numerical calculations.

[21] J. Guzowski, S. Jakiela, P. M. Korczyk, and P. Garstecki, Lab Chip 13, 4308 (2013).

[22] D. C. Morse and T. A. Witten, Europhys. Lett. 22, 549 (1993).

[23] S. H. Kim, H. Hwang, C. H. Lim, J. W. Shim, and S. M. Yang, Adv. Funct. Mater. 21, 1608 (2011).

[24] F. Malloggi, N. Pannacci, R. Attia, F. Monti, P. Mary, H. Willaime, P. Tabeling, B. Cabane, and P. Poncet, Langmuir 26, 2369 (2010).

[25] K. Ahn, C. Kerbage, T. P. Hunt, R. M. Westervelt, D. R. Link, and D. A. Weitz, Appl. Phys. Lett. 88, 024104 (2006).

[26] G. Villar, A. D. Graham, and H. Bayley, Science 340, 48 (2013).

[27] J. Guzowski, M. Tasinkevych, and S. Dietrich, Phys. Rev. E 84, 031401 (2011).

[28] J. Zhou, S. Long, Q. Wang, and A. D. Dinsmore, Science 312, 1631 (2006). 